\title{
Étude des caractères ampélographiques et phyllométriques des cépages mineurs du tel Algérien
}

\author{
Abderazak Hamama, Kaddour el Heit ${ }^{\mathrm{a}}$, Saida Meghezzi, Salima Sebki, Ounissa Agouazi et Mohend Said Cherfaoui \\ Laboratoires des Ressources Naturelles: Viticulture/Arboriculture, Faculté des Sciences et des Sciences Agronomiques, \\ Université Mouloud Mammeri, Tizi-Ouzou 15.000, Algérie
}

\begin{abstract}
Résumé. L’Algérie par ses potentialités pédoclimatiques, constitue un habitat naturel pour la vigne, en particulier la vigne autochtone dont la diversité génétique reste à mettre en valeur. Notre étude s'inscrit dans l'objectif de la caractérisation ampélographique de 21 cépages de vigne, appartenant à la collection de la station régionale de Benchicao (Médéa), en vue de leur identification et regroupement selon leurs similarités. Cette étude est entreprise en utilisant 85 descripteurs quantitatifs et 17 descripteurs qualitatifs établis par l'OIV. Une étude statistique est réalisée afin de mettre en évidence les paramètres les plus discriminants, à savoir, les angles et la profondeur des sinus latéraux par rapport aux longueurs des nervures. Cependant, l'analyse en composantes principales et la classification hiérarchique ont permis de regrouper les cépages en trois groupes plus au moins distincts, et a fait ressortir la présence de clones parmi les individus analysés. Cette diversité ne cesse de subir une érosion génétique continue, de ce fait leur sauvegarde devient plus qu'indispensable.
\end{abstract}

\begin{abstract}
Algeria by its potentialities of soil and climate constitutes a natural habitat for vines, especially the indigenous vine whose genetic diversity remains to valorize. The objective of our study is the ampelographic characterization of 21 cultivars of vines belonging to the collection of the regional station Benchicao (Medea), for identification and determination of the relationships between them and grouped according their similarities. This study is conducted using 85 quantitative descriptors and 17 qualitative descriptors established by the OIV. A statistical study was conducted to highlight the most discriminate parameters, namely, angles and depth of sinuses in relation to the lengths of veins. However, the principal component analysis and hierarchical classification have permitted to group the varieties in 3 Groups more or less distinct, which brought out the presence of clones among the individuals analyzed. This diversity is constantly undergoing genetic erosion, thus safeguarding it becomes indispensable.
\end{abstract}

\section{Introduction}

La culture de la vigne est bien enracinée dans les traditions des populations paysannes Maghrébines en générale et Algérienne en particulier. Depuis son extension à l'époque Phénicienne, Carthaginoise et Romaine, ces productions surtout en vin sont devenues une source de revenu à côté de la culture de l'olivier et du figuier [1]. En Algérie, les différentes mutations que subit l'espace agraire font que la diversité des ressources génétiques d'origine végétale se trouve dans une situation préoccupante de régression voire de disparition. Cette situation est à conjuguer aux effets de la mondialisation qui a causé une réduction de la diversité des cépages autochtones. Dans la perspective de cerner la diversité rencontrée au sein des cépages autochtones et de leur éventuelle conservation en collection, nous avons pris pour notre étude de caractérisation vingt et un (21) individus de Vitis vinifera L., selon différentes approches (ampélométriques et ampélographique).

Ce travail de caractérisation répond aux problèmes des risques de perte de la diversité génétique des Vitis vinifera L. Les cépages autochtones soumis à une érosion variétale

\footnotetext{
a e-mail : kaddy60023@yahoo.fr
}

progressive suite à la méconnaissance de leurs qualités agronomiques et technologiques.

\section{Matériels et méthodes}

Le matériel végétal est récolté dans la station régionale de l'institut technique de l'arboriculture et de la vigne de Benchicao (Médéa), les 21 accessions faisant l'objet de cette étude sont dénommées dans le tableau 1 :

Les ceps (12 répétitions par cépage) ont été disposés de manière aléatoire et plantés dans une parcelle expérimentale, cultivés en espalier et greffés sur le portegreffe $\mathrm{SO}_{4}$ et avec une taille en Guyot. Cette parcelle est très homogène en ce qui concerne la composition du sol et la topographie.

\subsection{Paramètres ampélographiques}

L'ensemble des caractères ampélographiques retenus dans notre étude selon la codification de l'O.I.V [11] pour la description des feuilles adultes et des bourgeonnements sont au nombre de 17 et ils sont : OIV 001, OIV 067, OIV 068, OIV 070, OIV 071, OIV 76, OIV 079, OIV 080,

This is an Open Access article distributed under the terms of the Creative Commons Attribution License 4.0, which permits unrestricted use, distribution, and reproduction in any medium, provided the original work is properly cited. 
Tableau 1. Cépages étudiés.

\begin{tabular}{|l|l|l|l|l|l|}
\hline $\mathbf{N}^{\circ}$ & Cépages & Origine & Aspect de la baie & Conduite & Exposition \\
\hline 1 & Ahchichane & Algérie & Verte & Espalier & Sud-Est \\
\hline 2 & Ahmar de Benchicao & Algérie & Noire & Espalier & Sud-Est \\
\hline 3 & Ahmar de Machtras III & Algérie & Rouge & Espalier & Sud-Est \\
\hline 4 & Ahmar de Mascara & Algérie & Rouge & Espalier & Sud-Est \\
\hline 5 & Ain El Bouma & Algérie & Blanche & Espalier & Sud-Est \\
\hline 6 & Amghar & Algérie & Noire & Espalier & Sud-Est \\
\hline 7 & Bezoul El Khadem & Algérie & Noire & Espalier & Sud-Est \\
\hline 8 & Chaouche & Algérie & Noire & Espalier & Sud-Est \\
\hline 9 & Cherchali & Algérie & Noire & Espalier & Sud-Est \\
\hline 10 & Ferrana Blanc & Algérie & Blanche & Espalier & Sud-Est \\
\hline 11 & Ferrana de Mascara & Algérie & Blanche & Espalier & Sud-Est \\
\hline 12 & Ferrana Noir & Algérie & Noire & Espalier & Sud-Est \\
\hline 13 & Muscat de Fondouk & Algérie & Blanche & Espalier & Sud-Est \\
\hline 14 & Muscat de Gustave & Algérie & Blanche & Espalier & Sud-Est \\
\hline 15 & Muscat d'El Adda & Algérie & Noire & Espalier & Sud-Est \\
\hline 16 & Sbaa El Tolba & Algérie & Blanche & Espalier & Sud-Est \\
\hline 17 & Tadelith & Algérie & Blanche & Espalier & Sud-Est \\
\hline 18 & Torki & Turquie & Noire & Espalier & Sud-Est \\
\hline 19 & Toutrisine & Algérie & Blanche & Espalier & Sud-Est \\
\hline 20 & Mokrani Blanc & Algérie & Blanche & Espalier & Sud-Est \\
\hline 21 & Mokrani Noir & Algérie & Noire & Espalier & Sud-Est \\
\hline
\end{tabular}

OIV 081-1, OIV 081-2, OIV 082*, OIV 83-1, OIV O83-2, OIV 093, OIV 094, OIV 223, OIV 225.

\subsection{Paramètres ampélométriques}

En se basant sur les stades phénologiques de Baggiolini (1952), les échantillonnages des feuilles adultes ont été réalisés entre la nouaison et la véraison. Nous avons sélectionné 15 feuilles par cépage. Plusieurs études démontrent qu'il s'agit d'un nombre d'échantillons amplement suffisant et représentatif ([2,3,5,7,9,11,15]). Les feuilles choisies se situaient entre $7^{\text {ème }}$ et $12^{\text {ème }}$ nœud, compté à partir de la base du rameau primaire suivant les recommandations de l'Organisation International de la Vigne et du Vin (2009).

Après un nettoyage avec de l'eau distillée et un préséchage avec du papier buvard, les feuilles sont pressées et mises en herbier suivant les recommandations de ([8,9]). Le séchage en herbier permet de stocker et de conserver les feuilles de chaque cépage plus longtemps jusqu'à l'opération de mesures ampélométriques.

Les feuilles séchées et bien aplanies sont prises en photos à l'aide d'un appareil à photos numérique de type Panasonic (Lumix DMC-FZ8) sur leur face inferieure et leur face supérieure.

La base des mesures, selon [4] et [8], repose sur 65 paramètres (longueurs des nervures, angles, dentelures, rapports des dentelures) mesurés en individualisant ceux correspondant au côté gauche et ceux du côté droit et ce, dans le but de mettre en évidence les éventuelles variations entre ces deux côtés de la feuille, complétés par vingt (20) indices de découpure du coté gauche et du coté droit (Fig. 1).

Les différentes mesures ont été effectuées par analyse numérique des images des feuilles prises pour cet effet, à l'aide du logiciel Image Tool 3 d'UTHSC (IT) version 3.00 (compdent.uthscsa.edu/dig/download.html) en utilisant un spécifique étalonnage, qui est un logiciel libre d'analyse et de traitement d'image [13].

\subsection{Analyses statistiques}

Nous avons effectué une analyse de variance, séparément pour chacun des paramètres à l'aide du logiciel XLSTAT 2012 et du logiciel Statbox 6.40 appuyée par une analyse factorielle des correspondances (A.F.C), une analyse en composantes principales (A.C.P) et une classification hiérarchisée (C.A.H), basée sur le degré de similitude.

\section{Résultats et discussion}

\subsection{Paramètres ampélographiques}

De étude descriptive des paramètres ampélographiques utilisés, il apparie que la forme du limbe (paramètre OIV 067) et des dents (paramètre OIV 076) ainsi la distribution de la pigmentation anthocyanique des nervures (paramètres OIV 070,071) donnent une dispersion plus au moins importante des cépages étudiés, ce qui corrobore avec les résultats obtenus par [4] et [15].

L'analyse factorielle des variables qualitatives montre que hormis (OIV-001) le degré d'ouverture de l'extrémité du bourgeonnement, (OIV-068) le nombre de lobes, (OIV076) la forme des dents, (OIV-083-1) la forme de la base des sinus latéraux supérieurs et (OIV-083-2) relative à la présence ou à l'absence de dents dans les sinus latéraux supérieurs, le reste des variables participe peu à la dispersion des individus selon les axes F1 et F2.

L'axe F1 détenant 34,76\% de l'inertie totale, oppose les cultivars : Ferrana Noire et Torki, qui se caractérisent par la même forme des dents (à deux cotés rectilignes), par le même nombre de lobe (cinq lobes), par la présence de 


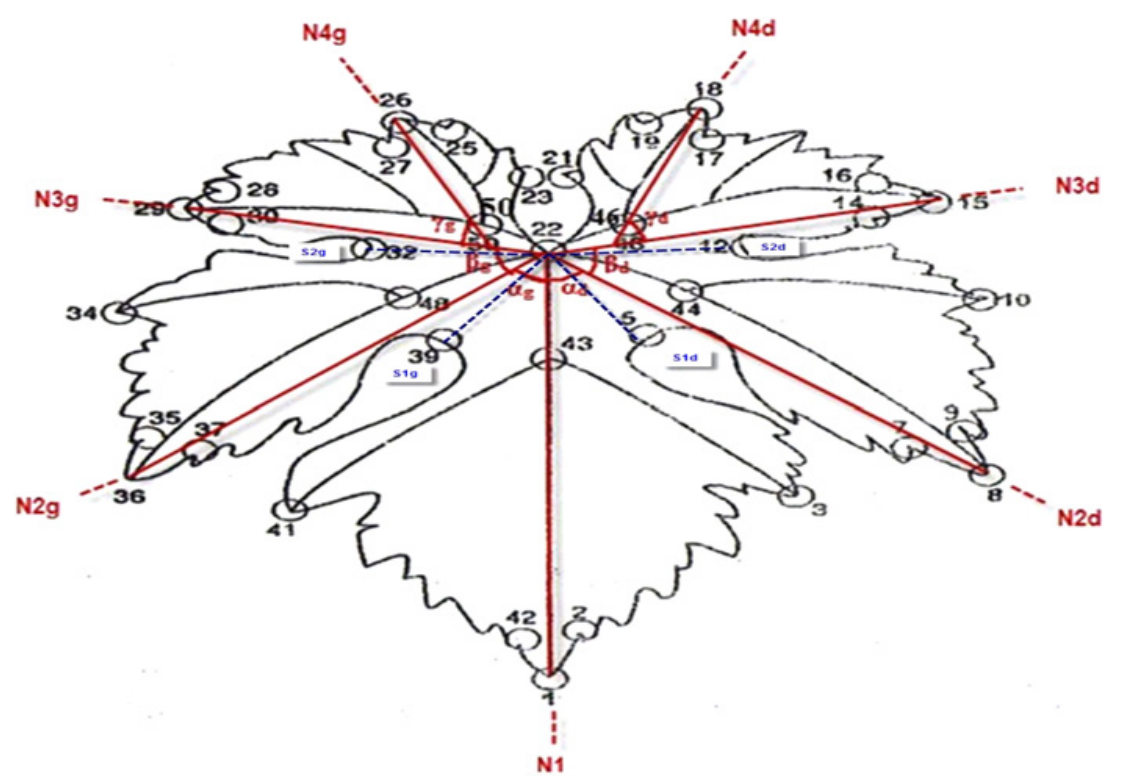

Figure 1. Schéma représentatif des points de la feuille pour calculer les paramètres ampélométriques ([12]) (Modifié). Figure 1. Schema of representative points of the sheet to calculate ampélométriques parameters ([12]) (Modified).

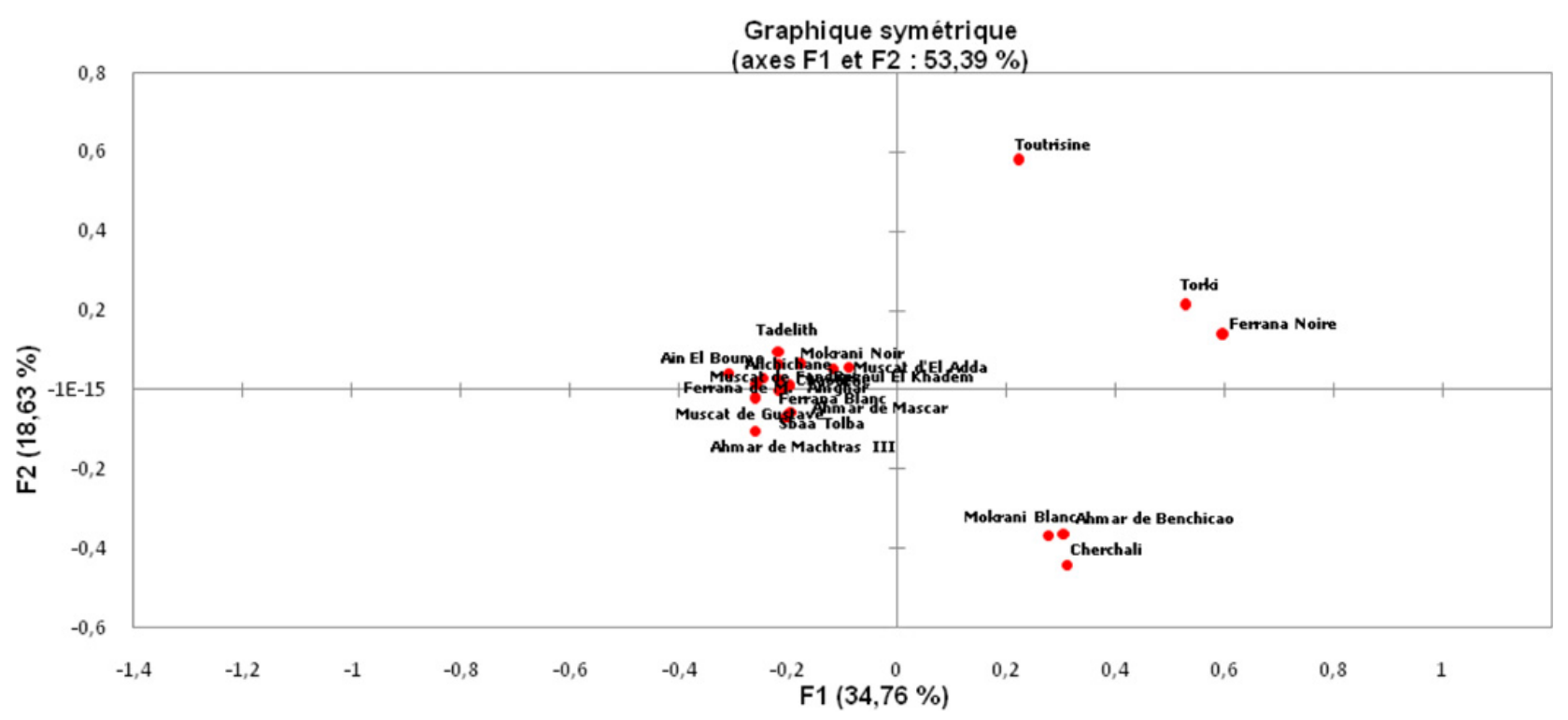

Figure 2. Représentation graphique des cépages étudiés en AFC.

Figure 2. Graphical representation of the varieties studied AFC.

dent dans les sinus latéraux supérieurs et par la présence de la pigmentation anthocyanique sur la face supérieure de la feuille aux cépages Ahchichane, Ahmar de Mascara, Ain El Bouma, Amghar, Ferrana de Mascara, Muscat de Fondouk et Muscat de Gustave se caractérisent par une extrémité de rameau complètement ouverte, par des baies de forme sphérique, par l'absence de pigmentation anthocyanique sur la face supérieure de la feuille et par l'absence de dent dans les sinus latéraux supérieurs.

Quant à l'axe F2 qui exprime 18,63\% de l'information totale. Il oppose le cépage Toutrisine qui se distingue des autres cépages étudiés par la présence de dents dans le sinus latéral supérieur de la feuille, également par des dents à deux cotés convexes et par la couleur rouge de sa baie aux cépages Ahmar de Benchicao, Cherchali et Mokrani Blanc présentant des sinus latéraux supérieurs en forme de $\langle\mathrm{U} »$, des feuilles ayant des dents à deux côtés rectilignes et sur lesquelles nous notons l'absence de dent dans les sinus latéraux supérieures.

Nous pouvons retenir de l'analyse que Toutrisine sort du lot individuellement des cépages par rapport à ces caractères ampélographiques particuliers cités en haut (Fig. 2).

\subsection{Classification ascendante hiérarchique (CAH)}

Des données Phyllométriques ont étés utilisées pour la classification hiérarchisée des cépages, réalisée au principe de la perte minimale d'inertie. Trois principaux groupes 


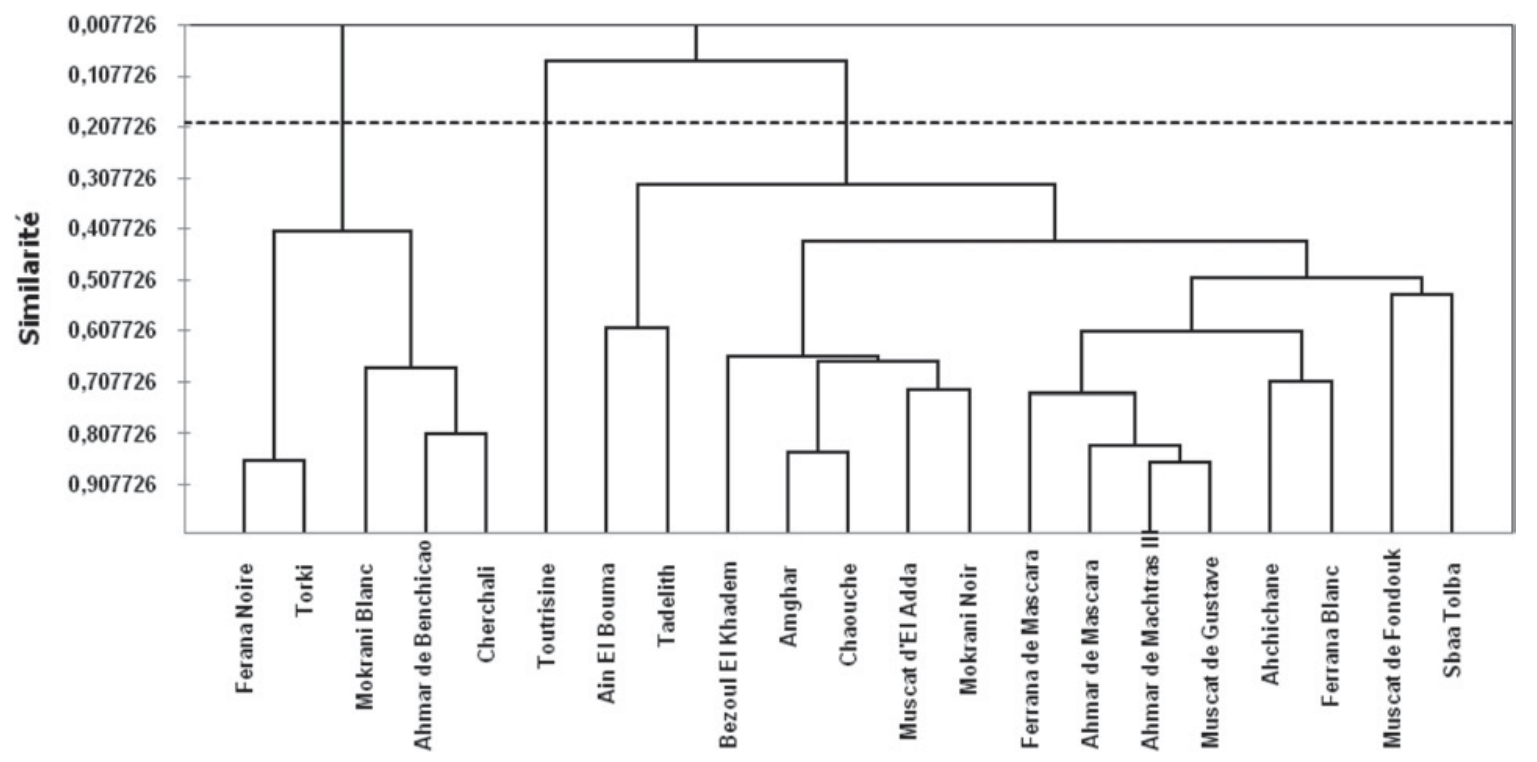

Figure 3. Classification ascendante hiérarchique.

Figure 3. Hierarchical clustering.

(A, B, C) sont représentés sure la Fig. 3, qui révèlent des similitudes entre les cépages étudiés : le premier comprend quinze cultivars. Les plus fortes similitudes sont enregistrées entre Ahmar de Machtras III et Muscat de Gustave $(0,87)$ en raison de la distribution de la pigmentation anthocyanique sur les deux faces de leurs feuilles et la forme de leurs dents, aussi chez Amghar et Chaouche $(0,83)$ vue la forme sphérique de leurs baies.

Le deuxième groupe divisé en deux sous groupes, il est composé de Torki et Ferrana noire avec une similarité de 0,85, d'Ahmar de Benchicao et Cherchali $(0,8)$ en plus de Mokrani blanc.

Les cultivars du deuxième groupe sont regroupés par rapport à la profondeur de leurs sinus latéraux supérieurs et par la forme de leurs baies sphériques.

Quant au troisième cluster il est représenté par l'unique cépage : Toutrisine se distinguant principalement des autres cépages étudiés, par la présence de pigmentation anthocyanique observée au point pétiolaire au niveau des deux faces inférieure et supérieure de la feuille. Deux autres caractères distinctifs sont à signaler, celui de la forme orbiculaire de la feuille et du chevauchement plus ou moins léger des sinus latéraux supérieurs de la feuille.

\subsection{Paramètres ampélométriques}

L'analyse de la variance (ANOVA) au seuil de $5 \%$ nous a permis de pouvoir comparer les moyennes observées dans le cas des 21 cépages étudiés et elle a montré qu'il existe des différences significatives pour la plupart des paramètres ampélométriques utilisés dans le cas de notre étude. Les variables quantitatives ont permis de différencier entre les individus étudiés avec une discrimination plus au moins apparente (Fig. 4).

Il ressort de l'ACP que le plan factoriel $(1 \times 2)$ obtient $55,09 \%$ de l'inertie totale. L'axe 1 englobant 34,64\% de l'information totale, il est défini positivement et principalement par les longueurs des nervures N3, N1, N2, et N4 (OIV 603, OIV 601, OIV 602, OIV 604) et par les mesures relatives aux sinus S1 et S2 (OIV 605, OIV 606). Il oppose les cépages Mokrani Blanc, Amghar et Ahmar de Mascara lesquels sont caractérisés par des nervures plus longues et des sinus les plus profonds (feuilles plus grandes, découpées) et Muscat de Fondouk, Ahmar de Benchicao et Muscat d'El Adda qui se caractérisent par des nervures plus courtes et des sinus moins profond (feuilles plus petites, moins découpées).

Quant à l'axe 2, qui représente 20,45\% de la part de l'information. Il est définit positivement par les angles (OIV-608, OIV-607, OIV-610 et OIV-609). Ces variables quantitatives sont signalées par [10] lesquelles confirment la part de ces angles dans l'élaboration de l'axe 2.

Les cultivars Ain El Bouma, Tadelith et Chaouche sont mieux représentés positivement par l'axe 2 . Ils se distinguent par des valeurs plus élevées pour les variables : beta (3.1.41), alpha (34.1.10), alpha (26.1.18), X1 (S1/N2) et $\mathrm{X} 5(\mathrm{~S} 1+\mathrm{S} 2 / \mathrm{N} 1+\mathrm{N} 2)$ de même pour : $\mathrm{S} 1$, alpha (32.1.12), $\mathrm{X} 3(\mathrm{~S} 1+\mathrm{S} 2 / \alpha+\beta)$ et $\mathrm{X} 6(\mathrm{~S} 1+\mathrm{S} 2 / \mathrm{N} 1+$ N2). A l'exception du cépage Chaouche lequel a obtenu des résultats moyens pour ces derniers paramètres.

De ce fait, ces cépages se caractérisent par rapport aux autres cépages étudiés par leurs feuilles plus larges et d'une découpure moins importante.

Du coté opposé de l'axe 2, les cépages : Cherchali, Toutrisine et Ferrana Noire se distinguent du reste des cépages étudiés par des feuilles moins larges et plus découpées.

En revanche, la variable $X_{5}=\left(S_{1}+S_{2}\right) /\left(N_{1}+N_{2}\right)$ qui contribue le plus à l'élaboration de l'axe 2 , est la variable qui donne le plus d'information par la dispersion des individus. Nos résultats sont similaires à ceux obtenus par (Martinez et al., 1997).

Globalement, AN (Largeur de la feuille), $\mathrm{X}_{5}=\left(\mathrm{S}_{1}+\right.$ $\left.\mathrm{S}_{2}\right) /\left(\mathrm{N}_{1}+\mathrm{N}_{2}\right)$ et les paramètres relatifs aux angles sont les paramètres foliaires qui contribués le plus à la différentiation entre les cultivars étudiés par rapport aux 


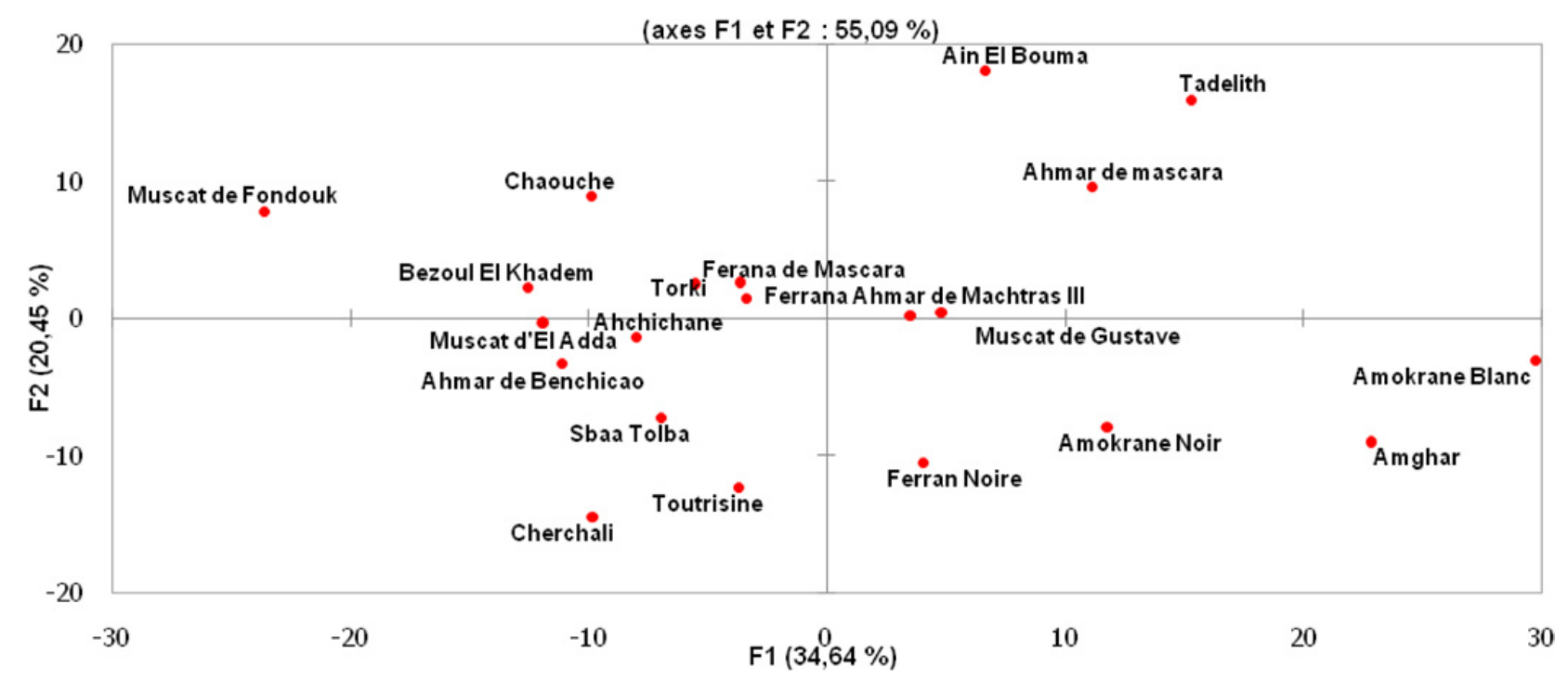

Figure 4. Représentation graphique des cépages étudiés en ACP.

Figure 4. Graphical representation of the varieties studied ACP.

autre variables soumises à l'étude, car selon ([10] et [3]), ils sont peu influençables par les conditions climatiques et pédologiques ainsi que les façons culturales.

\section{Conclusion}

En conclusion, notre travail de caractérisation par les deux approches ampélographique et ampélométrique qui sont complémentaires, constitue un outil très utile pour la différentiation entre les cépages étudiés.

L'étude ampélographique des différents organes des individus étudiés a montré l'existence d'un polymorphisme foliaire et de différences morphologiques au sein des cultivars étudiés.

L'approche ampélographique a été affinée par une analyse factorielle des correspondances (A.F.C) et une classification hiérarchisées fournissant plus de détails sur la classification des cultivars, d'où la distinction de Toutrisine distingué par le nombre de lobes que porte sa feuille, lesquelles se chiffre à sept lobes bien distincts. Cette distinction apparait aussi avec la présence d'une dent au niveau du sinus pétiolaire.

Même si l'ampélographie classique est encore la méthode la plus utilisée de façon pratique par un grand nombre de personnes surtout les spécialistes de la vigne, l'Ampélométrie vient compléter ces données [4].

A travers les résultats des analyses statistiques des différents paramètres ampélométriques étudiés, il apparaît que les cépages autochtones sont différents phénotypiquement.

En effet, les plus grandes différences sont enregistrées entre les cultivars : Mokrani Blanc et Muscat de Fondouk par rapport à la longueur des nervures, aussi entre Cherchali et Tadelith par rapport à la découpure de leurs feuilles.

De même, les analyses ont permis de mettre en évidence le paramètre $X_{5}=\left(S_{1 \mathrm{~g}+} \mathrm{S}_{2 \mathrm{~g}}\right) /(\mathrm{N} 1+\mathrm{N} 2)$ relatif à la découpure de la feuille, parmi les paramètres foliaires qui détient le plus fort pouvoir discriminant par rapport aux autres paramètres soumis à l'étude.

L'approche que nous avons utilisée appréciable si nous tenons compte des appréciations formulées par [4]. Selon lui, l'utilisation des clés de détermination des cépages à partir des mesures ou des données ampélométriques, nous semble valable pour une zone ou une région donnée délimitée avec un nombre de cépages limité, car il faut savoir que les composantes du climat ont beaucoup d'influence sur la vigueur ce qui influence considérablement sur les paramètres étudiés. Par conséquent, il n'est pas possible d'envisager l'élaboration d'une clé valide et universelle.

Bien que de nouvelles méthodes de caractérisation plus précises ([5]), comme la caractérisation par le biais des marqueurs microsatellites présente un intérêt fort utile lorsqu'il s'agit d'établir les parentés entre cépage cultivés et sauvages et de mettre fin aux problèmes de synonymies entre cépages.

\section{Références}

[1] AOUF M.B., 1972. La reconversion-reconstitution du vignoble algérien. In. La vigne et le vin : CIEHAM (Options Méditerranéennes), pp. 65-67

[2] Branas J., 1974. Viticulture. Imp. Déhan, Montpellier. France

[3] Boursiquot J.M., Vignau L. et Boulet J.C., 1989. Ricerche sull'utilizzazione dell'ampelometria. Riv. Vitic. Eno., 1, 37-52

[4] Cid-Alvarz N., Boursiquot J.M., Saa-Otero M.P. et Martinez L.R., 1994. Différenciation des cépages autochtones du Nord-Ouest de l'Espagne (Galice) et élaboration d'une clé de détermination basée sur l'Ampélométrie Jour. Int. Scie. Vigne et vin. Vol. 28 No.1: pp. 1-17

[5] Detweiller E., 1991. Preliminary minimal descriptor list of grapevine varieties. Institut für Rebenzüchtung Geilweilerhof. Siebeldingen. 12, pp. 10-17 
[6] Galet P., 2000a Dictionnaire Encyclopédique des cépages, $762 \mathrm{p}$.

[7] Galet P., 2000b Précis de viticulture. $7^{\text {éme }}$ édition. Dehan. Montpellier, $561 \mathrm{p}$.

[8] Martinez M.C. et Grenan S., 1999. A graphic reconstruction method of an average vine leaf. Agronomie 19: pp. 491-507

[9] Martinez M.C., Boursiquot J.M., Grenan S. et Boidron R., 1997. Étude ampélométriques des feuilles adultes de somaclones de cv. De Grenache N. Can. Bot. 75: pp. 333-345

[10] Martinez De Toda F et SANCHA J.C., 1997. Caractérisation ampélographique des cultivars blancs de Vitis vinifera L. conservés en Rioja. Bulletin de l'OIV (793-794), pp. 221-234

[11] OIV 2009: Office internationale de la vigne et du vin. Le code des caractères descriptifs des variétés et espèces de Vitis.Ed. Dedon, Paris

[12] Romani L., Legido J.L., Saa M.P. and Cid Alvarez N., 1993. Design and updating of a computer system for the ampelometric caracterization of grape vine varieties. J. Wine Research, 4, $\mathrm{n}^{\mathrm{o}} 3$, pp. 197-203

[13] Rotaru L., 2005. The application of discriminant factorial analysis for the establishing phenotypical homogeniti of Europeo-Americanes rootstock. Universitatea de Ştiinţe Agricole şi Medicină Veterinară Iaşi. Hortic.: pp. 287-292

[14] Santiago J.L., Boso S., Gago P., Villaverde V. and Martinez M.C., 2007. Molecular and ampelographic characterisation of Vitis vinífera L. Albariño, Savagnin, Blanc and Caiño Blanco. Spanish Journal of Agricultural Research 5(3): pp. 333-340

[15] Sefc K.M., Lefort F., Grando M.S., Scott K.D., Steinkellner H. and Thomas M.R., 2001. Microsatellite markers for grapevine: a state of the art. In: Roubelakis-Angelakis K.A. (eds), molecular biology and biotechnology of grapevine, Kluwer Academic Publishers. Amsterdam. pp. 433-463 